\title{
Smart delivery with nanofactories
}

Researchers engineer biological 'nanofactories' that can trigger a quorum sensing response in bacteria.

One goal of the rapidly growing nanotechnology field is to make nanoscale devices that can interact with biological systems. William Bentley of the University of Maryland has in particular been interested in developing

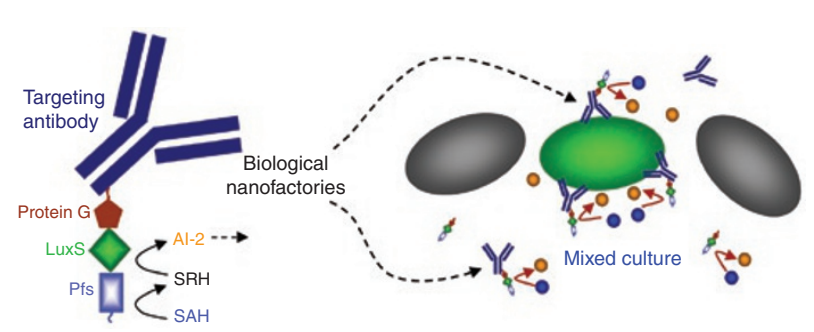

A biological nanofactory to trigger quorum sensing. Assembly of nanofactories that convert SAH to AI-2 (left). The nanofactories target only the bacteria of interest in a mixed culture (right). Reprinted from Nature Nanotechnology.

technologies to understand and manipulate biological signaling. His group recently reported the assembly of protein-based 'nanofactories' that can stimulate quorum sensing in bacteria (Fernandes et al., 2010).

A biological nanofactory, first described in 2007 by Philip LeDuc of Carnegie Mellon University, Michael Wong of Rice University and an assembly of colleagues (LeDuc et al., 2007), is a nanoscale machine consisting of various modules that can target a particular type of cell, sense a raw material at that site and synthesize a new, useful molecule from that raw material. The scientists proposed the use of nanofactories as a novel form of drug treatment-for example, the nanofactory could be targeted to a tumor, where it would sense a naturally produced compound and then convert this compound into a tumorkilling agent, on site.

Being interested in signaling, Bentley's group sought to make a nanofactory that could target specific bacteria and synthesize and deliver autoinducer-2 (AI-2), a 'universal' bacterial quorum sensing signaling molecule. They designed a sensing and synthesis module consisting of the AI-2 synthases $S$-adenosylhomocysteine nucleosidase (Pfs) and $S$-ribosylhomocysteinase (LuxS). As the targeting module, they selected an immunoglobulin gamma (IgG) antibody specific to a bacterial species of interest. To assemble the modules, they fused the AI-2 synthases to protein $\mathrm{G}$, which binds to the IgG. "By putting in the protein $G$, you can take advantage of biology's small-scale assembly technology," explains Bentley, emphasizing that this was one of their key developments. This flexible self-assembly module allows them to load the nanofactory with any IgG of interest.

The assembled nanofactory localizes to the relevant bacteria and converts the substrate $S$-(5'-deoxyadenosine$\left.5^{\prime}\right)$-L-homocysteine (SAH) to AI-2, right where the researchers want it. Bentley's group was able to generate an enhanced quorum sensing response in $\operatorname{luxS}$ null mutant strains of Salmonella typhimurium and Escherichia coli that express $\beta$-galactosidase via an AI-2-responsive promoter. They could selectively target either one or the other species in a co-culture by changing the IgG. And notably, they could also make the two species, which do not normally communicate, 'talk' to each other.

The group is currently building on their success in triggering quorum sensing to explore how to control bacterial biofilm formation. These nanofactories could potentially be used in novel antimicrobial treatments that act on the communication networks between bacteria. In addition to 'smart' drug delivery, another application is in vivo imaging. "You could develop a smart contrast agent that also carries a drug or drug synthesis capability," notes Bentley.

And unlike many nanotechnology tools proposed to interface with biological systems, Bentley's nanofactories are all proteinbased. So for drug-treatment applications, for example, "you don't have issues with liver toxicity and so on," he says. Perhaps someday, biological nanofactories will be zooming around our bodies, keeping us healthy.

\section{Allison Doerr}

\section{RESEARCH PAPERS}

Fernandes, R. et al. Engineered biological nanofactories trigger quorum sensing response in targeted bacteria. Nat. Nanotechnol. advance online publication (17 January 2010).

LeDuc, P.R. et al. Towards an in vivo biologically inspired nanofactory. Nat. Nanotechnol. 2, 3-7 (2007). 